\title{
Article
}

\section{On parametric equivalence, isomorphism and uniqueness: Cycle related graphs}

\author{
J. Kok ${ }^{1,2, *}$ and J. Shiny ${ }^{3}$ \\ 1 Independent Mathematics Researcher, City of Tshwane, South Africa. \\ 2 Visiting Faculty at CHRIST (Deemed to be a University), Bangalore, India. \\ 3 Mathematics Research Center, Mary Matha Arts and Science College, Kerala, India. \\ * Correspondence: jacotype@gmail.com; johan.kok@christuniversity.in; Tel.: +27646547285
}

Academic Editor: Aisha Javed

Received: 28 January 2021; Accepted: 15 April 2021; Published: 30 April 2021.

Abstract: This furthers the notions of parametric equivalence, isomorphism and uniqueness in graphs. Results for certain cycle related graphs are presented. Avenues for further research are also suggested.

Keywords: Parametric equivalence; Parametric isomorphism; Parametric uniqueness.

MSC: 05C12; 05C38; 05C69.

\section{Introduction}

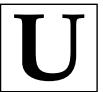
nless stated otherwise, graphs will be finite, undirected and connected simple graphs. A shortest path having end vertices $u$ and $v$ is denoted by, $u-v_{(\text {in } G)}$. If $d_{G}(u, v) \geq 2$ then a vertex $w$ on $u-v_{(\text {in } G)}$, $w \neq u, w \neq v$ is called an internal vertex on $u-v_{(i n G)}$. When the context is clear the notation such as $d_{G}(u, v)$, $\operatorname{deg}_{G}(v)$ can be abbreviated to $d(u, v), \operatorname{deg}(v)$ and so on. Good references to important concepts, notation and graph parameters can be found in [1-3].

The notions of parametric equivalence, isomorphism and uniqueness had been introduced in [4]. For ease of reference we recall from [4] as follows: Let $\rho$ denote some minimum or maximum graph parameter related to subsets $V(G)$ of graph $G$. Vertex subsets $X$ and $Y$ is said to be parametric equivalent or $\rho$-equivalent if and only if both $X, Y$ satisfy the parametric conditions of $\rho$. This relation is denoted by $X \equiv_{\rho} Y$. Furthermore, if $X \equiv \rho Y$ and the induced graphs $\langle V(G) \backslash X\rangle \cong\langle V(G) \backslash Y\rangle$ then $X$ and $Y$ are said to be parametric isomorphic. This isomorphic relation is denoted by $X \cong \rho$. Let all possible vertex subsets of graph $G$ which satisfy $\rho$ be $X_{1}, X_{2}, X_{3}, \ldots, X_{k}$. If $X_{1} \cong \rho X_{2} \cong \rho X_{3} \cong \rho \cdots \rho X_{k}$ then $X_{i}, 1 \leq i \leq k$ are said to be parametric unique or $\rho$-unique. The graph $G$ is said to have a parametric unique or $\rho$-unique solution (or parametric unique $\rho$-set). If $G$ has a unique (exactly one) $\rho$-set $X$, then $X$ is a parametric unique $\rho$-set.

This paper furthers the introductory research presented in [4].

\section{Confluence in graphs}

Shiny et al., [5] introduced the concept of a confluence set (a subset of vertices) of a graph $G$, also see [6] for results on certain derivative graphs. Recall that for a non-complete graph $G$, a non-empty subset $\mathcal{X} \subseteq V(G)$ is said to be a confluence set if for every unordered pair $\{u, v\}$ of distinct vertices (if such exist) in $V(G) \backslash \mathcal{X}$ for which $d_{G}(u, v) \geq 2$ there exists at least one $u-v_{(i n G)}$ with at least one internal vertex, $w \in \mathcal{X}$. Also a vertex $u \in \mathcal{X}$ is called a confluence vertex of $G$. A minimal confluence set $\mathcal{X}$ (also called a $\zeta$-set) has no proper subset which is a confluence set of $G$. The cardinality of a minimum confluence set is called the confluence number of $G$ and is denoted by $\zeta(G)$. A minimal confluence set is denoted by $\mathcal{C}$. To distinguish between different graphs the notation $\mathcal{C}_{G}$ may be used for a minimum confluence set of $G$. We recall two important results from [4]. We remind that for a complete graph the confluence number is 0 hence, $\mathcal{C}_{K_{n}}=\varnothing, n \geq 1$.

Proposition 1. [4] A path $P_{n}$ has a parametric unique $\zeta$-set if and only if $n=1,2$ or $n=4+3 i$ or $n=5+3 i, i=0,1,2, \ldots$ 
Proposition 2. [4] $A$ cycle $C_{n}$ has a parametric unique $\zeta$-set if and only if $n=3,4$ or $n=5+3 i$ or $n=6+3 i$, $i=0,1,2, \ldots$

\subsection{Cycle related graphs}

Henceforth, a cycle $C_{n}, n \geq 3$ of order $n$ has the vertex set $V\left(C_{n}\right)=\left\{v_{i}: i=1,2,3, \ldots, n\right\}$.

(a) A wheel graph (simply, a wheel) $W_{n}$ is obtained from a cycle $C_{n}, n \geq 3$ with an additional central vertex $v_{0}$ and the additional edges $v_{0} v_{1}, 1 \leq i \leq n$. The cycle is called the rim and the edges $v_{0} v_{i}, 1 \leq i \leq n$ are called spokes. Alternatively, $W_{n}=C_{n}+K_{1}$ and $V\left(K_{1}\right)=\left\{v_{0}\right\}$.

Proposition 3. A wheel graph $W_{n}$ has a parametric unique $\zeta$-set.

Proof. Since $W_{3}$ is complete the result is trivial. For $n \geq 4$ the distance $d\left(v_{i}, v_{j}\right) \leq 2$ for all distinct pairs. For $i, j \neq 0$ and $v_{i}$ not adjacent to $v_{j}$ there exists a 3-path (or 2-distance path) with $v_{0}$ the internal vertex. Hence, the unique $\zeta$-set is $\left\{v_{0}\right\}$, therefore parametric unique.

(b) A helm graph $H_{n}$ is obtained from a wheel graph $W_{n}$ by adding a pendent vertex (or leaf) $u_{i}$ to each rim vertex $v_{i}$.

Proposition 4. (a) The helm graph $\mathrm{H}_{3}$ does not have a parametric unique $\zeta$-set.

(b) A helm graph $H_{n}, n \geq 4$ has a parametric unique $\zeta$-set.

Proof. (a) Consider $H_{3}$. Clearly and without loss of generality the sets $X_{1}=\left\{v_{0}, v_{1}, v_{2}\right\}, X_{2}=\left\{v_{1}, v_{2}, v_{3}\right\}$ and $X_{3}=\left\{v_{1}, v_{2}, u_{3}\right\}$ are all minimal confluence sets. Hence $\zeta\left(H_{3}\right) \leq 3$. It is easy to verify that no 2 -vertex subset is a confluence set. Thus, $\zeta\left(H_{3}\right)>2$. Also, $\left\langle V\left(H_{3}\right) \backslash X_{1}\right\rangle \nRightarrow\left\langle V\left(H_{3}\right) \backslash X_{2}\right\rangle$. Therefore $H_{3}$ does not have a parametric unique $\zeta$-set. The aforesaid follows in essence from the fact that $H_{3}$ is complete. Therefore, it is not necessary for $v_{0}$ to be in all $\zeta$-sets.

(b) For $H_{n}, n \geq 4$ the distance $d\left(u_{i}, u_{i+1}\right)=3$ hence a rim vertex is required. The distance $d\left(u_{i}, u_{i+2}\right)=5$ hence the vertex $v_{0}$ will suffice along the 5-path $u_{i} v_{i} v_{0} v_{i+2} u_{i+2}$. By symmetry considerations and therefore up to isomorphism and without loss of generality we have two subcases.

Subcase 1. If $n$ is even the set $X_{1}=\left\{v_{0}, v_{1}, v_{3}, v_{5}, \ldots, v_{n-1}\right\}$ is a $\zeta$-set and clearly $H_{n}$ has a parametric unique $\zeta$-set.

Subcase 2. If $n$ is odd the sets $X_{1}=\left\{v_{0}, v_{1}, v_{3}, v_{5}, \ldots, v_{n-1}\right\}$ and $X_{2}=\left\{v_{0}, v_{1}, v_{3}, v_{5}, \ldots, v_{n-2}, v_{n}\right\}$ are a $\zeta$-sets. Clearly $\left\langle V\left(H_{n}\right) \backslash X_{1}\right\rangle \cong\left\langle V\left(H_{n}\right) \backslash X_{1}\right\rangle$. Thus $H_{n}$ has a parametric unique $\zeta$-set.

As a direct consequence of the proof of Proposition 4, we get the next corollary.

Corollary 1. A helm graph has $\zeta\left(H_{n}\right)=\left\lceil\frac{n}{2}\right\rceil+1$.

(c) A flower graph $F l_{n}$ is obtained from a helm graph $H_{n}$ by adding the edges $v_{0} u_{i}, 1 \leq i \leq n$.

Proposition 5. A flower graph $\mathrm{Fl}_{n}$ has a parametric unique $\zeta$-set.

Proof. The result follows by similar reasoning as in the proof of Proposition 3.

As a direct consequence of Proposition 5, we get the next corollary.

Corollary 2. A flower graph has $\zeta\left(F l_{n}\right)=1$.

(d) A closed helm graph $H_{n}^{c}$ is obtained from a helm graph $H_{n}$ by completing a cycle, $C_{n}^{\prime}=u_{1} u_{2} u_{3} \cdots u_{n} u_{1}$ on the leafs of $H_{n}$.

Proposition 6. (a) A closed helm graph $H_{n}^{c}$ for $n=4$ or $n$ is odd does not have a parametric unique $\zeta$-set.

(b) A closed helm graph $H_{n}^{c}, n \geq 6$ and even, has a parametric unique $\zeta$-set. 
Proof. It is easy to verify that all distance paths such that $d\left(u_{i}, u_{j}\right) \leq 3$ are paths on $C_{n}^{\prime}$. Also, for $u_{i}, u_{j} \in \mathcal{C}_{C_{n}^{\prime}}$ we have $d\left(u_{i}, u_{j}\right) \leq 3$. It follows that $\mathcal{C}_{C_{n}^{\prime}} \subseteq \mathcal{C}_{H_{n}^{c}}$.

(a) By similar reasoning to that in the proof of Proposition 4(a) it follows that $H_{3}^{c}$ and $H_{4}^{c}$ do not have a unique $\zeta$-set.

From the set $X_{1}=\left\{v_{i}: u_{i} \notin \mathcal{C}_{C_{n}^{\prime}}\right\} \cup\left\{v_{0}\right\}$ it is possible to select a minimum confluence set in respect of the spanning subgraph $H_{n}$ say set $X_{2}$. The set $\mathcal{C}_{H_{n}^{c}}=\mathcal{C}_{C_{n}^{\prime}} \cup X_{2}$ is a minimum confluence set.

Subcase (a)(1). Since by symmetry the choice of say, $X_{2}$ can be fixed, For $n \geq 5$ and odd, the choice of $\mathcal{C}_{C_{n}^{\prime}}$ can rotate such that $\left\langle V\left(H_{n}^{c}\right) \backslash \mathcal{C}_{H_{n}^{c}}\right\rangle$ does not remain isomorphic.

(b) By similar reasoning $X_{2}$ can be fixed. However, for $n \geq 6$ and even and by symmetry properties of $C_{n}^{\prime}$ all choices of $\mathcal{C}_{C_{n}^{\prime}}$ yield isomorphic $\left\langle V\left(H_{n}^{c}\right) \backslash \mathcal{C}_{H_{n}^{c}}\right\rangle$.

As a direct consequence of the proof of Proposition 6, we get the next corollary.

Corollary 3. A closed helm graph has $\zeta\left(H_{n}^{c}\right)=\left\lceil\frac{n}{2}\right\rceil+1$.

(e) A gear graph $G_{n}$ is obtain from a wheel graph $W_{n}$ by inserting a vertex $u_{i}$ on the edge $v_{i} v_{i+1}$ and $n+1 \equiv 1$. Note that $G_{n}$ has $2 n+1$ vertices and $3 n$ edges. The rim is now called a boundary cycle denoted by $C^{b}\left(G_{n}\right)$.

Proposition 7. (a) G3 has a parametric unique $\zeta$-set.

(b) A gear graph $G_{n}$ and $n \geq 5$ is odd does not have a parametric unique $\zeta$-set.

(c) A gear graph $G_{n}$ and $n \geq 4$ is even has a parametric unique $\zeta$-set.

Proof. (a) For $G_{3}$ it follows easily that up to isomorphism the $\zeta$-set $\left\{u_{1}, v_{3}\right\}$ is unique.

(b) The inner-area enclosed by the cycle $C_{2 n}^{\prime}=v_{1} u_{1} v_{2} u_{2} \cdots v_{n} u_{n} v_{1}$ can be partitioned into $n$ planar areas, each enclosed by a $C_{4}$. For all pairs $v_{i}, v_{j}$ it is necessary and sufficient that $v_{0} \in \zeta$-set. Let $n \geq 5$ be odd. Without loss of generality, an optimal minimal confluence set is given by $X_{1}=\left\{v_{0}, u_{1}, u_{3}, \ldots, u_{n-2}, u_{n-1}\right\}$ or $X_{2}=$ $\left\{v_{0}, u_{1}, u_{3}, \ldots, u_{n-2}, v_{n}\right\}$ or $X_{3}=\left\{v_{0}, u_{1}, u_{3}, \ldots, u_{n-2}, u_{n}\right\}$. Hence, $\zeta\left(G_{n}\right) \leq\left\lceil\frac{2 n}{4}\right\rceil+1=\left\lceil\frac{n}{2}\right\rceil+1$. Because the boundary cycle $C^{b}\left(G_{n}\right)$ has $\zeta\left(C^{b}\left(G_{n}\right)\right)=\left\lceil\frac{2 n}{3}\right\rceil$ it follows that $\zeta\left(G_{n}\right) \geq\left\lceil\frac{2 n}{3}\right\rceil$. However for $n$ is odd, $\left\lceil\frac{2 n}{3}\right\rceil=\left\lceil\frac{n}{2}\right\rceil+1$. Since,

$$
\left\langle V\left(G_{n}\right) \backslash X_{1}\right\rangle \varsubsetneqq\left\langle V\left(G_{n}\right) \backslash X_{2}\right\rangle .
$$

It follows that a gear graph $G_{n}$ does not have a parametric unique $\zeta$-set for $n$ is odd.

(c) For $n \geq 4$ and even, reasoning similar to that in (b) show that up to isomorphism the $\zeta$-set $X_{1}=$ $\left\{v_{0}, u_{1}, u_{3}, \ldots, u_{n-2}, u_{n-1}\right\}$ is unique. Reasoning in respect of bounds on $\zeta\left(G_{n}\right)$ similar to that in (a) settles the result.

As a direct consequence of the proof of Proposition 7, we get the next corollary.

Corollary 4. The gear graph $G_{3}$ has $\zeta\left(G_{3}\right)=2$. A gear graph of order $n \geq 4$ has $\zeta\left(G_{n}\right)=\left\lceil\frac{n}{2}\right\rceil+1$.

(f) A sun graph $S_{n}^{\boxplus} n \geq 3$ is obtained by taking the complete graph $K_{n}$ on the vertices $v_{1}, v_{2}, v_{3}, \ldots, v_{n}$ together the isolated vertices $u_{i}, 1 \leq i \leq n$ and adding the edges $v_{i} u_{i}, u_{i} v_{i+1}$ and $n+1 \equiv 1$. The boundary cycle of a sun graph is the cycle $C^{b}\left(S_{n}^{\bigotimes}\right)=v_{1} u_{1} v_{2} u_{2} v_{3} u_{3} \cdots u_{n} v_{1}$.

Proposition 8. A sun graph $S_{n}^{\bigotimes}, n \geq 3$ has a parametric unique $\zeta$-set if and only if $C^{b}\left(S_{n}^{\bigotimes}\right)$ is of order $n=3 i, i=$ $1,2,3, \ldots$

Proof. Since all pairs $v_{i}, v_{j}$ are adjacent it suffices to only consider a $\zeta$-set of $C^{b}\left(S_{n}^{\otimes}\right)$. Since $\operatorname{deg}\left(u_{i}\right)=2$ and $\operatorname{deg}\left(v_{j}\right)=3$ any $\zeta$-set must be graphically symmetrical for a sun graph to have a parametric unique $\zeta$-set. A graphically symmetrical $\zeta$-set means that, measured along the boundary cycle, $\min \left\{d\left(v_{j}, u_{k}\right): v_{j}, u_{k} \in \zeta\right.$-set $\}=$ 3. It implies that $n=3 i, i=1,2,3, \ldots$. 
The converse follows from the fact that sun graphs with $C^{b}\left(S_{n}^{\boxplus}\right)$ of order $n \neq 3 i, i=1,2,3, \ldots$ do not have graphically symmetrical $\zeta$-sets of even order.

Note that if a sun graph has a parametric unique $\zeta$-set then $\zeta\left(S_{n}^{\boxplus}\right)$ is even. Furthermore, as a direct consequence of the proof of Proposition 8 , we get the next corollary.

Corollary 5. A sun graph has $\zeta\left(S_{n}^{\bowtie}\right)=\left\lceil\frac{2 n}{3}\right\rceil$.

(g) A sunflower graph $S_{n}^{\circledast}, n \geq 3$ is obtained by taking the wheel graph $W_{n}$ together the isolated vertices $u_{i}$, $1 \leq i \leq n$ and adding the edges $v_{i} u_{i}, u_{i} v_{i+1}$ and $n+1 \equiv 1$. The boundary cycle of a sun graph is the cycle $C^{b}\left(S_{n}^{\circledast}\right)=v_{1} u_{1} v_{2} u_{2} v_{3} u_{3} \cdots u_{n} v_{1}$.

Proposition 9. A sunflower graph $S_{n}^{\circledast}, n \geq 3$ does not have a parametric unique $\zeta$-set.

Proof. For all pairs $v_{i}, v_{j}$ it is sufficient that $v_{0} \in \zeta$-set. Thereafter any $\zeta$-set $X_{1}$ in respect of $C^{b}\left(S_{n}^{\circledast}\right)$ is required to obtain $\mathcal{C}_{S_{n}^{\circledast}}=X_{1} \cup\left\{v_{0}\right\}$. It implies that $\zeta\left(S_{n}^{\circledast}\right)=n$. In turn, the aforesaid confluence number permits that say, $X_{2}=\left\{v_{1}, v_{2}, v_{3}, \ldots, v_{n}\right\}$ or $X_{3}=\left\{v_{1}, v_{2}, v_{3}, \ldots, v_{n-1}, u_{n-1}\right\}$ are $\zeta$-sets. Since, $\left\langle V\left(S_{n}^{\circledast}\right) \backslash X_{1}\right\rangle \varsubsetneqq\left\langle V\left(S_{n}^{\circledast}\right) \backslash X_{2}\right\rangle \varsubsetneqq$ $\left\langle V\left(S_{n}^{\circledast}\right) \backslash X_{3}\right\rangle$ the result follows.

As a direct consequence of the proof of Proposition 9, we get the next corollary.

Corollary 6. A sunflower graph has $\zeta\left(S_{n}^{\circledast}\right)=n$.

(h) A sunlet graph $S_{n}^{\ominus}, n \geq 3$ is obtained by taking cycle $C_{n}$ together the isolated vertices $u_{i}, 1 \leq i \leq n$ and adding the pendent edges $v_{i} u_{i}$.

Proposition 10. A sunlet graph $S_{n}^{\ominus}, n \geq 3$ has a parametric unique $\zeta$-set.

Proof. Case 1. Let $n \geq 3$ and odd. Without loss of generality and by isomorphism, it is easy to verify that the sets $X_{1}=\left\{v_{1}, v_{3}, v_{5}, \ldots, v_{n}\right\}$ and $X_{2}=\left\{v_{1}, v_{3}, v_{5}, \ldots, v_{n-2}, v_{n-1}\right\}$ are $\zeta$-sets. Furthermore, up to isomorphism those are the only distinguishable $\zeta$-sets. Since,

$$
\left\langle( V ( S _ { n } ^ { \ominus } ) \backslash X _ { 1 } \rangle \cong \left\langle\left(V\left(S_{n}^{\ominus}\right) \backslash X_{2}\right\rangle,\right.\right.
$$

the result follows for $n \geq 3$ and odd.

Case 2. By similar reasoning as in Case 1 the result follows for $n \geq 4$ and even.

As a direct consequence of the proof of Proposition 10, we get the next corollary.

Corollary 7. A sunlet graph has $\zeta\left(S_{n}^{\ominus}\right)=\left\lceil\frac{n}{2}\right\rceil$.

(i) A circular ladder (or prism graph) $L_{n}^{\circ}, n \geq 3$ is obtained by taking two cycles of equal order $n$. Label as, $C_{n}^{1}=v_{1} v_{2} v_{3} \cdots v_{n} v_{1}$ and $C_{n}^{2}=u_{1} u_{2} u_{3} \cdots u_{n} u_{1}$. Add the edges $v_{i} u_{i}, 1 \leq i \leq n$. A circular ladder can be viewed as $H_{n}^{c}-v_{0}$.

Proposition 11. A circular ladder graph $L_{n}^{\circ}$ has a parametric unique $\zeta$-set if and only if $n=4$ or $n=3$ i for $i=2,3,4, \ldots$.

Proof. Part 1. For $n=4, X_{i}=\left\{u_{i}, v_{j}\right\}, i=1,2,3,4, j \in\{1,2,3,4\}$ such that $d\left(u_{i}, v_{j}\right)=3$, are the minimum confluence sets for $L_{4}^{\circ}$. Since $\left\langle V\left(L_{4}^{\circ}\right) \backslash X_{i}\right\rangle$ are $C_{6}$ for $i=1,2,3,4$, we have the result for $n=4$.

In a circular ladder graph $L_{n}^{\circ}, n \neq 4$ there are $n$ copies of $C_{4}=v_{i} u_{i} u_{i+1} v_{i+1}$. For each $C_{4}=v_{i} u_{i} u_{i+1} v_{i+1}$, at least one of the vertices $v_{i}, u_{i}, u_{i+1}, v_{i+1}$ belongs to every minimum confluence set of $L_{n}^{\circ}$.

Part 2. For $n=3, X_{1}=\left\{v_{1}, v_{2}\right\}$ and $X_{2}=\left\{v_{1}, u_{2}\right\}$ are two minimum confluence set for $L_{3}^{\circ}$. However, $\left\langle V\left(L_{3}^{\circ}\right) \backslash X_{1}\right\rangle$ and $\left\langle V\left(L_{3}^{\circ}\right) \backslash X_{2}\right\rangle$ are not isomorphic. Hence $L_{3}^{\circ}$ has no unique parametric set.

Part 3. For $n=3 i, i=2,3, .$. , let $\mathcal{C}_{C_{n}}\left(v_{i}\right)$ be a minimum confluence set of $C_{n}$ starting from $v_{i}$ and $C_{C_{n}^{\prime}}\left(u_{j}\right)$ be a minimum confluence set of $C_{n}^{\prime}$ starting from $u_{j}$. Then for $i \neq j, X_{i j}=\mathcal{C}_{C_{n}}\left(v_{i}\right) \cup \mathcal{C}_{C_{n}^{\prime}}\left(u_{j}\right)$ is a minimum confluence 
set for $L_{n}^{\circ}$ and $\left\langle V\left(L_{n}^{\circ}\right) \backslash X_{i j}\right\rangle$ consists of $\frac{n}{3}$ copies of $P_{3}$. Hence the result for $n=3 i, i=2,3, \ldots$

Part 4. If $n \equiv 2(\bmod 3)$. Let $X_{1}$ be the minimum confluence set for $L_{n}^{\circ}$ such that $u_{i}, u_{i+2}, v_{i+1} \in X_{1}$ and let $X_{2}$ be the minimum confluence set for $L_{n}^{\circ}$ such that $u_{i}, u_{i+2}, v_{i} \in X_{2}$. Then $\left\langle V\left(L_{n}^{\circ}\right) \backslash X_{1}\right\rangle$ and $\left\langle V\left(L_{n}^{\circ}\right) \backslash X_{2}\right\rangle$ are not isomorphic. Hence $L_{n}^{\circ}$ has no parametric unique set if $n_{\geq 5} \equiv 2(\bmod 3)$.

By a similar argument we have to prove that $L_{n}^{\circ}$ has no parametric unique set if $n_{\geq 7} \equiv 1(\bmod 3)$.

Since all $n \in \mathbb{N}_{\geq 3}$ have been accounted for the 'if' has been settled.

For all valid cases the converse, 'only if', follows through reasoning by contradiction.

Corollary 8. A circular ladder has,

$$
\zeta\left(L_{n}^{\circ}\right)= \begin{cases}2, & \text { if } n=4 ; \\ 2\left\lceil\frac{n}{3}\right\rceil, & \text { if } n=3 \text { or } n \geq 5 .\end{cases}
$$

Proof. The result is a consequence of the proof of Proposition 11. The exception lies in the fact that $L_{4}^{\circ}$ has $5=n_{=4}+1$ cycles $C_{4}$ to account for. All other $L_{n_{* 4}}^{\circ}$ have $n$ cycles $C_{4}$ to account for.

Observe that the confluence number of a circular ladder is always even.

(j) A tadpole graph $T(m, n), m \geq 3, n \geq 1$ is obtained from a cycle $C_{m}=v_{1} v_{2} v_{3} \cdots v_{m} v_{1}$ and a path $P_{n}=$ $u_{1} u_{2} u_{3} \cdots u_{n}$ by adding an edge between an end-vertex of $P_{n}$ and a vertex of $C_{m}$. The new edge is also called a bridge.

Proposition 12. A tadpole graph $T(m, n), m \geq 3, n \geq 1$ :

(a) Tadpole graphs $T(3, n), n \geq 1$ have a parametric unique $\zeta$-set if and only if $n=3 i, i=1,2,3, \ldots$

(b) Tadpole graphs $T(4,1), T(4,2)$ have a parametric unique $\zeta$-sets.

(c) Tadpole graphs $T(5,1)$ does not have a parametric unique $\zeta$-set and $T(5,2)$ has.

(d) Tadpole graphs $T(m, 1), T(m, 2), m \geq 6$ have a parametric unique $\zeta$-set if and only if $m=6+3 i, i=0,1,2, \ldots$

(e) Tadpole graphs $T(m, n), m \geq 4$ and $n \geq 3$ have a parametric unique $\zeta$-set if and only if both the cycle $C_{m}$ and the path $P_{n}$ have parametric unique $\zeta$-sets.

(f) All other tadpole graphs as excluded through (a) to (f) do not have a parametric unique $\zeta$-set.

Proof. (a) The tadpole graphs $T(3, n), n \geq 1$ does not have a parametric unique $\zeta$-set for $P_{1}, P_{2}$ (straightforward).

Subcase (a)(1). For $n+2=5+3 i, i=0,1,2, \ldots$ the $\zeta$-set of $P_{n+2}$ is unique hence, $T(3, n)$ has a parametric unique $\zeta$-set.

Subcase (a)(2). For $n+2=6+3 i, i=0,1,2, \ldots$ the $\zeta$-set of $P_{n+2}$ is not parametric unique hence, $T(3, n)$ does not have a parametric unique $\zeta$-set.

Subcase (a)(3). For $n+2=7+3 i, i=0,1,2, \ldots$ the $\zeta$-set of $P_{n+2}$ is parametric unique. However, since some $\zeta$-sets may contain vertex $v_{j}$ of the bridge the tadpole $T(3, n)$ does not have a parametric unique $\zeta$-set.

All tadpoles $T(3, n), n \geq 1$ have been accounted for because,

$$
\mathbb{N}=\{1,2\} \cup\{3+3 i: i=0,1,2, \ldots\} \cup\{4+3 i: i=0,1,2, \ldots\} \cup\{5+3 i: i=0,1,2, \ldots\} .
$$

(b) The tadpole graphs $T(4, n), n \geq 1$ have a parametric unique $\zeta$-set for $P_{1}, P_{2}$. It follows from the fact that a bridge vertex say, $v_{i}$ has to be in any $\zeta$-set.

Subcases $n+2=5+3 i, n+2=6+3 i$ and $n+2=7+3 i, i=0,1,2, \ldots$ will be settled in (d) and (e) below.

(c) The tadpole graphs $T(5, n), n \geq 1$ does not have a parametric unique $\zeta$-set for $P_{1}$ bacause it is easy to verify that an end-vertex of the bridge need not be in all $\zeta$-sets. However for $P_{2}$ the tadpole has a parametric unique $\zeta$-set. It follows from the fact that a bridge vertex say, $v_{i}$ has to be in any $\zeta$-set.

Subcases $n+2=5+3 i, n+2=6+3 i$ and $n+2=7+3 i, i=0,1,2, \ldots$ will be settled in (d) and (e) below.

(d) The tadpoles $T(m, 1), T(m, 2), m \geq 6$ do not require that vertices $u_{1}$ and /or $u_{2}$ to necessarily be in a $\zeta$-set. Hence, all $\zeta$-sets of cycle $C_{m}$ which contain a vertex of the bridge suffice to be $\zeta$-sets of the tadpoles. Therefore has a parametric unique $\zeta$-set if and only if $C_{m}$ has a unique $\zeta$-set. Therefore, if and only if $m=6+3 i, i=0,1,2, \ldots$ The converse follows easily by contradiction. 
(e) Finally, for a tadpole $T(m, n), m \geq 4$ and $n \geq 3$ and both the cycle $C_{m}$ and the path $P_{n}$ have parametric unique $\zeta$-sets, it is easy to verify that the $\zeta$-sets of the tadpole all contain a vertex $v_{j}$ of the bridge. Therefore the tadpole has a parametric $\zeta$-set. Else, it is always possible to find a $\zeta$-set of the tadpole which contains a vertex $v_{j}$ which is on the bridge and another $\zeta$-set which does not. Therefore, such tadpoles do not have a parametric unique $\zeta$-set. Hence, the tadpoles $T(m, n), m \geq 4$ and $n \geq 3$ have a parametric unique $\zeta$-set if and only if both $C_{m}$ and $P_{n}$ have parametric unique $\zeta$-sets.

(f) All other tadpole graphs which were excluded through reasoning of proof, (a) to (e) do not have a parametric unique $\zeta$-set.

(k) A lollipop graph $L^{\otimes}(m, n), m \geq 3, n \geq 1$ is obtained from a complete graph $K_{m}$ and a path $P_{n}$ by adding a bridge between an end-vertex of $P_{n}$ and a vertex of $C_{m}$.

Proposition 13. A lollipop graph $L^{\otimes}(m, n), m \geq 3, n \geq 1$ has a parametric unique $\zeta$-set if and only if $n=3 i, i=$ $1,2,3, \ldots$

Proof. The proof follows directly from the proof of Proposition 12(a).

(1) A generalized barbell graph $B(n, m), n, m \geq 3$ is obtained from two complete graph $K_{n}, K_{m}$ and adding a bridge.

Proposition 14. A generalized barbell graph $B(n, m), n, m \geq 3$ has a parametric unique $\zeta$-set if and only if $n=m$.

Proof. Let $K_{n}$ be on vertices $v_{1}, v_{2}, v_{3}, \ldots, v_{n}$ and $K_{m}$ on vertices $u_{1}, u_{2}, u_{3}, \ldots, u_{m}$. For any pair $v_{i} u_{j}$ and edge $v_{i} u_{j}$ not the bridge, the distance $d\left(v_{i}, u_{j}\right)=2$ or 3 . Therefore any vertex of the bridge yields a $\zeta$-set. Without loss of generality let the $\zeta$-set be $\left\{v_{k}\right\}$. It follows that $\left\langle V(B(n, m)) \backslash\left\{v_{k}\right\}\right\rangle \cong K_{n-1} \cup K_{m}$. Hence, $B(n, m)$ has a parametric unique $\zeta$-set if and only if $n=m$.

\section{Conclusion}

The study of cycle related graphs has not exhausted. Note that for those cycle related graphs which do not have a parametric unique $\zeta$-set the proof by contradiction can be utilized well.

The idea of combined parametric conditions remains open. Note that the parametric conditions will be ordered pairs. For example, the path $P_{3}=v_{1} v_{2} v_{3}$ has a unique minimum dominating set i.e. the $\gamma$-set $X_{1}=\left\{v_{2}\right\}$. Since $X_{1}$ is also a $\zeta$-set of $P_{3}$ the set is said to be a parametric unique $(\gamma, \zeta)$-set. However, since $X_{1}$ per se is not a parametric unique $\zeta$-set, it cannot be said to be a parametric unique $(\zeta, \gamma)$-set. On the other hand for a star $S_{1, n}, n \geq 3$ the set $X_{1}=\left\{v_{0}\right\}$ is both a parametric $(\gamma, \zeta)$-set and a parametric unique $(\zeta, \gamma)$-set. Studying such parametric combinations for say parameters $\rho_{1}(G)$ and $\rho_{2}(G)$ requires that, $\rho_{1}(G)=\rho_{2}(G)$.

Conjecture 1. If graph $G$ has a pendent vertex then $G$ has a unique $\zeta$-set if and only no $\zeta$-set exists which contains a pendent vertex.

A strict proof of Corollary 8 through mathematical induction is an interesting exercise for the reader.

Acknowledgments: The authors would like to thank the anonymous referees for their constructive comments, which helped to improve on the elegance of this paper.

Author Contributions: All authors contributed equally to the writing of this paper. All authors read and approved the final manuscript.

Conflicts of Interest: "The authors declare no conflict of interest."

\section{References}

[1] Bondy, J.A., \& Murty, U.S.R. (1976). Graph Theory with Applications. Macmillan Press, London.

[2] Harary, F. (1969). Graph Theory. Addison-Wesley, Reading MA.

[3] West, B. (1996). Introduction to Graph Theory. Prentice-Hall, Upper Saddle River.

[4] Kok, J.,\& Shiny, J. (2021). On parametric equivalent, isomorphic and unique sets. Open Journal of Discrete Applied Mathematics, 4(1), 19-24. 
[5] Shiny, J., Kok, J., \& Ajitha, V. Confluence number of graphs. Communicated.

[6] Kok, J., \& Shiny, J. Confluence number of certain derivative graphs. Communicated.

(C) 2021 by the authors; licensee PSRP, Lahore, Pakistan. This article is an open access article distributed under the terms and conditions of the Creative Commons Attribution (CC-BY) license (http://creativecommons.org/licenses/by/4.0/). 